\title{
Universal critical behavior and the transition temperature in (2+1)-flavor QCD
}

\author{
Christian Schmidt for the HotQCD Collaboration ${ }^{1}$ \\ Frankfurt Institute for Advanced Studies, J.W.Goethe Universität Frankfurt, D-60438 Frankfurt, Germany \\ GSI Helmholtzzentrum für Schwerionenforschung, Planckstr. 1, D-64291 Darmstadt, Germany
}

\begin{abstract}
We discuss the universal critical behavior in (2+1)-flavor QCD by analyzing lattice data from improved staggered fermions generated by the HotQCD Collaboration. We present recent results from two different lattice discretizations and various lattice spacings $\left(N_{\tau}=6,8,12\right)$ at fixed physical strange quark mass $\left(m_{s}\right)$ but varying light quark mass $\left(m_{l}\right)$. We find that the chiral order-parameter, i.e. the chiral condensate, shows the expected universal scaling that is associated with the critical point in the chiral limit already for light quark masses $m_{l} / m_{s} \lesssim 0.05$. From an analysis of the disconnected chiral susceptibility we estimate a preliminary value of the QCD transition temperature.
\end{abstract}

Keywords: Lattice Simulations, Improved Actions, Quark-Gluon-Plasma, Phase Transitions, Critical Behavior PACS: $12.38 . \mathrm{Gc}, 25.75 . \mathrm{Nq}$

\section{INTRODUCTION}

For almost 3 decades, lattice QCD is providing us with valuable information on QCD thermodynamics. Only recently, however, the numerical calculations arrive at a precision level, where controlled continuum extrapolations become possible. Moreover, our understanding of lattice cut-off effects resulting from the discretization of the QCD Lagrangian developed over the years.

We will present here results from two improved staggered actions: asqtad, which has been widely used for finite temperature calculations in the past [1] and the recently introduced highly improved staggered quark (HISQ) action [2]. Apart from different treatments in the gauge part, these actions mainly differ in their strategies for the reduction of the flavor symmetry breaking, which is inherent to the staggered fermion formulation. The HISQ action has been shown to offer the most superior degree of improvement in the flavor symmetry sector $[2,3]$. In the following we will refer to the action that incorporates the HISQ improvement program in the fermion sector together with a tree-level Symanzik improvement in the gauge part as HISQ/tree.

At sufficiently high temperature, QCD undergoes a transition form hadronic to quark degrees of freedom, which is a true phase transition only in the limit of massless quarks. This transition, driven by the restauration of the chiral symmetry, is of second order in the chi-

\footnotetext{
${ }^{1}$ HotQCD Collaboration members are: A. Bazavov, T. Bhattacharya, M. Cheng, N.H. Christ, C. DeTar, S. Gottlieb, R. Gupta, P. Hegde, U.M. Heller, C. Jung, F. Karsch, E. Laermann, L. Levkova, C. Miao, R.D. Mawhinney, S. Mukherjee, P. Petreczky, D. Renfrew, C. Schmidt, R.A. Soltz, W. Soeldner, R. Sugar, D. Toussaint, W. Unger, P. Vranas
}

ral limit. All thermodynamic quantities that can be derived as derivatives from the QCD partition function are thus subject to a universal critical behavior. In the following we will analyze the universal critical behavior of the chiral condensate, which is a true order-parameter in the chiral limit. Furthermore we estimate the transition temperature from the disconnected part of the chiral susceptibility.

The lattice data has been generated by the HotQCD collaboration [4] with the above mentioned actions on lattices with temporal extent of $N_{\tau}=6,8$ and for the asqtad action with $N_{\tau}=12$. This translates into lattice spacings $a$ of roughly $a=0.16,0.12$ and $0.08 \mathrm{fm}$ (at $T=$ $200 \mathrm{MeV}$ ). Throughout all calculations the strange quark mass $m_{s}$ was fixed to its physical value, whereas we have varied the light quark mass $m_{l}$ in the range $m_{l} / m_{s}=$ $0.05-0.2$. The physical value of light to strange quark mass is approximately given by $m_{l} / m_{s}=0.037$.

\section{UNIVERSAL SCALING}

The QCD phase transition in the chiral limit of 2-flavor QCD is expected to be in the $O(4)$ universality class [5]. For a sufficiently large strange quark mass this is expected to be the same in $(2+1)$-flavor QCD. The staggered fermion formulation on the lattice brakes chiral symmetry. For each fixed lattice spacing one is only left with a $O(2)$ symmetry. The $O(4)$ symmetry will, of course, be restored in the continuum limit.

In order to analyze the universal critical behavior that arises in the chiral limit we have to arrange the free energy (and its derivatives) in terms of the relevant scaling variables. In general one separates two kinds of contri- 

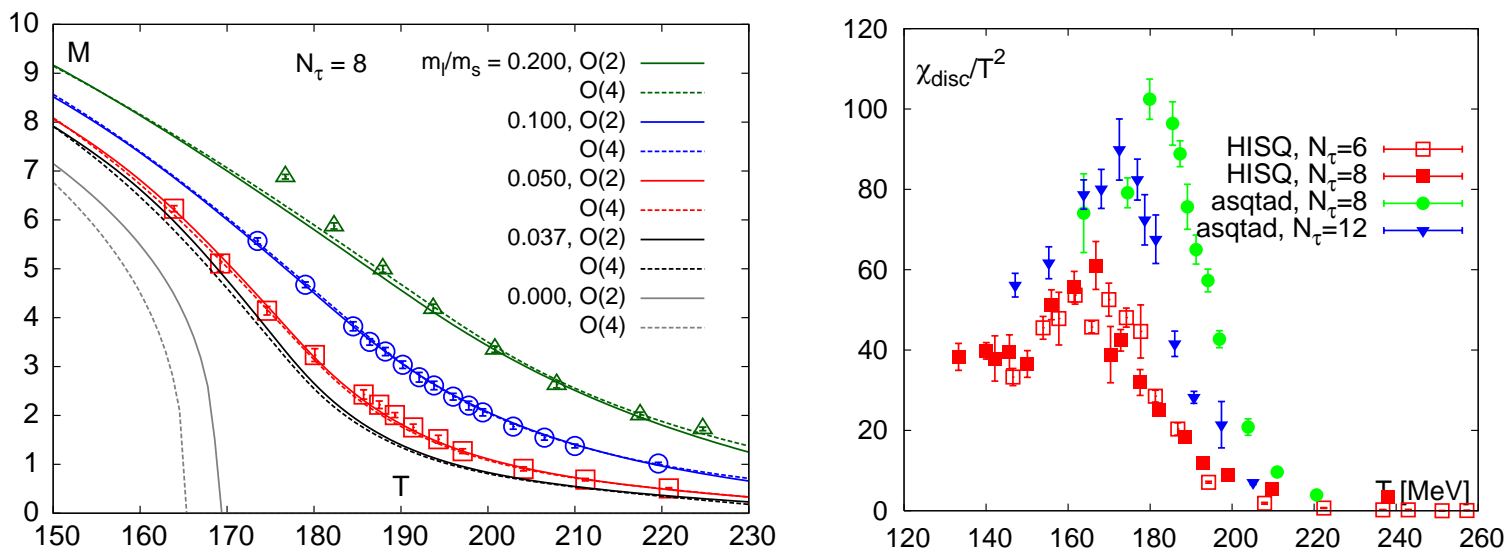

FIGURE 1. Left: Chiral order-parameter as defined in Eq. (3) for different quark masses, plotted as a function of temperature. Simulations have been performed with the asqtad action obtained on $N_{\tau}=8$ lattices. Right: Disconnected part of the chiral susceptibility for $m_{l} / m_{s}=0.05$. Compared are results from two different lattice actions and different lattice spacings.

butions to the free energy density, a part $\left(f_{s}\right)$ that will generate singularities in higher order derivatives of the partition function and a regular part $\left(f_{r}\right)$, we define

$$
f\left(T, m_{l}, m_{s}\right)=f_{s}\left(T, m_{l}, m_{s}\right)+f_{r}\left(T, m_{l}, m_{s}\right) .
$$

Although $f_{s}$ depends on many parameters of the QCD Lagrangian, the universal behavior can be expressed in only two relevant scaling variables $\mathrm{t}$ and $\mathrm{h}$, that control deviations from criticality at $(t, h)=(0,0)$. The scaling variables are up to some normalization factors $t_{0}, h_{0}$ given by the reduced temperature $t=\left(T-T_{c}\right) /\left(t_{0} T_{c}\right)$ and the symmetry braking parameter, which we will define here as $h=m_{l} /\left(h_{0} m_{s}\right)$. The universal critical behavior of the order parameter, $M \sim \partial f / \partial m_{l}$, is controlled by a scaling function $f_{G}$ that arises from the singular part of the free energy density after taking a derivative with respect to the light quark mass. We make use of the fact that $f_{s}$ is a homogeneous function of its arguments. $f_{G}$ (and $f_{s}$ ) can thus be written as a function of the single scaling variable $z=t / h^{1 / \delta \beta}$, where $\delta$ and $\beta$ are critical exponents of the three dimensional $O(N)$ universality class. In addition we also consider the leading oder contribution coming from the regular term of the free energy. We finally arrive at

$$
M=h^{1 / \delta} f_{G}(z)+a_{t} t h+b_{1} h
$$

The scaling function $f_{G}$ is well known from spin-model simulations and easily accessible via, e.g., the implicit parameterization given in [6]. On the right hand site of Eq. (2) we are left with 5 unknown parameters, $t_{0}, h_{0}, T_{c}, a_{t}, b_{1}$, which have to be determined through a fit to the lattice data. Out of these, $T_{c}$ is the only one that does not depend on the particular definition of the order-parameter $M$. All of them are, however, cut-off de- pendent and needed to be extrapolated to the continuum limit.

On the lattice the chiral order parameter (chiral condensate) is always finite but contains contributions that diverge in the continuum limit, i.e. it requires renormalization and in particular an additive and multiplicative renormalization. In order to remove at least the multiplicative renomalization factor already on the lattice we consider here the following definition of the orderparameter

$$
M=m_{s}\langle\bar{\psi} \psi\rangle_{l} / T^{4} .
$$

In Fig. 1 (left) we show the chiral oder-parameter as defined in Eq. (3) from simulations with the asqtad action obtained on lattices with temporal extend $N_{\tau}=8$ and light quark masses ranging from $m_{l} / m_{s}=0.05-0.2$. Also shown are fits to the $O(2)$ (solid curves) and $O(4)$ (dashed curves) scaling functions. In the fits we have omitted the data set with the largest quark mass $m_{l} / m_{s}=$ 0.2 . For masses $m_{l} / m_{s} \lesssim 0.1$ the fit describes the lattice data rather good in the entire temperature range we have considered. This coincides with finding obtained with the p4 action on $N_{\tau}=4$ [7] and $N_{\tau}=8$ [8]. Using the fits, we can extrapolate the chiral order-parameter to the physical mass $\left(m_{l} / m_{s}=0.037\right)$ and the chiral limit, as also indicated in the Fig. 1 (left). The main difference between the fits manifests in the chiral limit. We obtain values for $T_{c}$ ranging from $T_{c} \approx(165-170) \mathrm{MeV}$ (obtained for $N_{\tau}=8$ with the asqtad action).

\section{THE CRITICAL TEMPERATURE}

It is the ultimate goal and a valuable input for many phenomenological calculations, e.g. in heavy ion phenomenology, to obtain a continuum extrapolated value 


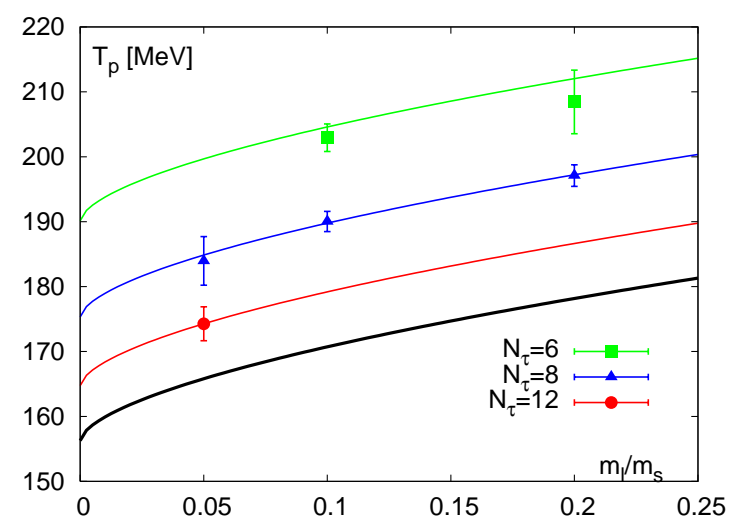

FIGURE 2. Transition temperature as obtained from the peak position of the disconnected part of the chiral susceptibility for different light quark masses and lattice spacings from simulations with the asqtad action. Also show is a combined chiral and continuum fit with the Ansatz given in Eq. 5.

for $T_{c}$. The chiral susceptibility is traditionally used to determine the QCD transition temperature. This quantity exhibits a peak as plotted as a function of temperature, which position can be associated with the QCD transition. Note that at nonzero light quark mass the QCD transition is a smooth crossover rather then a genuine phase transition of second Order. In this case the corresponding pseudo-critical temperature is not unique; it depends on the observable used to define it. All definitions will, however, lead to the same value for $T_{c}$ in the chiral and continuum limit.

The chiral susceptibility which has a connected and disconnected part is defined as the mass derivative of the order-parameter

$$
\chi \equiv \frac{T}{V} \frac{\partial}{\partial m_{l}}\langle\bar{\psi} \psi\rangle_{l} \equiv \chi_{\mathrm{dis}}+\chi_{\mathrm{con}} .
$$

In Fig. 1 (right) we plot the disconnected part of the chiral susceptibility as obtianed for light quark mass $m_{l} / m_{s}=0.05$ but different lattice spacings. We compare results from two different lattice actions: asqtad and HISQ/tree. We find that the cut-off effects are visible in the peak hight and peak position. They are considerably smaller in case of the HISQ/tree action.

From the scaling function for the chiral condensate, Eq. (2), the scaling of the chiral susceptibility can be easily extracted. It is governed by the function $f_{G}^{\prime}(z) \equiv$ $\mathrm{d} f_{G} / \mathrm{d} z$. To obtain a consistent picture it is desirable to perform a similar analysis as presented for the orderparameter in the previous section. This should lead to the same non-universal normalizations of the scaling fields as well as to the same value for $T_{c}$ in the chiral limit. At the moment, however, we restrict ourselves to an analysis of the peak positions $T_{p}$ of the disconnected part $\chi_{\text {dis }}$.
From Eq. (2) we obtain the fit Ansatz

$$
T_{p}\left(m_{l}, N_{\tau}\right)=T_{c}+b\left(m_{l} / m_{s}\right)^{d}+c N_{\tau}^{-2} .
$$

Here we allow for a generic cut-off dependence of the form $1 / N_{\tau}^{2}$ in addition to the mass dependence coming from the scaling Ansatz. We thus have three fit parameters, $T_{c}, b, c$. The exponent $d$ is fixed by universal scaling as $d=1 / \beta \delta$.

In Fig. 2 we show the peak positions $T_{p}$ from $\chi_{\text {dis }}$ as obtained by simulations with the asqtad action for different quark masses and lattice spacings. The values and their errors have been estimated by two different asymmetric fits to the peak [9]. In addition we show a combined chiral and continuum extrapolation based on Eq. (5). The fit works reasonably well and we obtain as a preliminary result for the transition temperature at physical masses:

$$
T_{p}\left(0.037 m_{s}, \infty\right)=164(6) \mathrm{MeV} .
$$

Here the error summarizes our estimate of the statistical and systematical error.

\section{ACKNOWLEDGMENTS}

This work has been supported in part by contracts DEAC02-98CH10886 and DE-FC02-06ER-41439 with the U.S. Department of Energy and contract 0555397 with the National Science Foundation. The numerical calculations have been performed using USQCD resources at Fermilab and JLab, the BlueGene/L at the New York Center for Computational Sciences (NYCCS), and the BlueGene/L at the Jülich Supercomputing Center. CS has partially been supported through the Helmholtz International Center for FAIR which is part of the Hessian LOEWE initiative.

\section{REFERENCES}

1. for an overview see e.g.: C.E. DeTar, PoS LATTICE2008, 001 (2008).

2. E. Follana et al. [HPQCD collaboration and UKQCD collaboration], Phys. Rev. D 75054502 (2007).

3. A. Bazavov et al. [MILC collaboration], Phys. Rev. D 82, 074501 (2010);

A. Bazavov and P. Petreczky, PoS Lattice 2010169.

4. A. Bazavov et al. [HotQCD collaboration], in preparation.

5. R. Pisarski and F. Wilczek, Phys. Rev. D 29, 338 (1984).

6. J. Engels, S. Holtmann, T. Mendes and T. Schulze, Phys. Lett. B 514, 299 (2001).

7. S. Ejiri et al., Phys. Rev. D 80, 094505 (2009).

8. O. Kaczmarek et al., arXiv:1011.3130 [hep-lat]; C. Schmidt and S. Mukherjee, PoS Lattice 2010 214;

9. W. Söldner, PoS Lattice 2010215. 